\title{
Las paradojas cognitivas de las tecnologías de la información
}

\author{
Umberto Roncoroni Osio \\ (Universidad de Lima, Perú)
}

Recibido: 16/12/2014

Aprobado: 4/6/2015

\begin{abstract}
Resumen. En este trabajo se hace un análisis de las dinámicas de producción y consumo cultural de la sociedad de la información en relación con los medios digitales. Ciertas propiedades de los medios de comunicación y de las TIC pueden producir, como muchos críticos han comenzado a señalar, una suerte de involución cognitiva que se expresa en nuevas formas de ignorancia y conformismo; se trata de procesos muy peligrosos porque actúan desapercibidos en el optimismo acrítico que caracteriza las utopías digitales. Lo que se intentará demostrar es que estos problemas no dependen de la técnica, sino de los principios y de los fundamentos ontológicos que rigen nuestra relación con la tecnología.
\end{abstract}

Palabras clave: comunicación / conformismo / creatividad / industria cultural / sobreproducción / tecnologías de la información

\section{The Cognitive Paradoxes of Information Technologies}

AвSTRACT. In this paper we analyze the cultural and commercial dynamics of the information society in relation to digital media. As many scholars point out, certain features of communication media can produce a type of cognitive regression that is expressed in new forms of ignorance and conformism. These processes are very dangerous as they go frequently unnoticed under the noncritical optimism that characterizes digital utopias. We aim at demonstrating that these problems do not depend on technology itself but on the principles and foundations that govern our relationship with technology

Key words: communication / conformism / creativity / information technologies / overproduction / show business 


\section{Introducción}

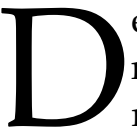
e las utopías de la posmodernidad, la sociedad del conocimiento es la más mediática y popular; estaría constituida por una nueva dimensión, sustentada por las tecnologías de la información, llena de oportunidades increíbles, como la new economy, en la que prosperan start ups, proyectos indie y makers por doquier, donde se goza de nuevas herramientas intelectuales, como la inteligencia artificial o el arte generativo. Un contexto, pues, que parece inaugurar una época de creatividad, innovación, democracia, libertad y abundancia cultural y empresarial.

Pero la lógica económica y la naturaleza acelerada de esta utopía generan, en la realidad, sobreproducción material e intelectual, exceso de datos y de comunicación, saturación y contaminación; se trata de fenómenos, como muchos autores comienzan a señalar (Kurz, 2003; Vega, 2007; Carr, 2008; Morozov, 2013) que ya revelan una serie de contradicciones del sistema y que hacen dudar de los beneficios culturales y sociales aclamados por los utopistas digitales.

El problema del exceso y de la sobreproducción, pues, no afecta solamente los dominios tecnocientíficos, como descubrió Vannevar Bush (1945). Este autor pensó solucionar el problema tecnológicamente, mediante una hipertextualidad ante litteram, pero los efectos no son cuantitativos, sino cualitativos; por ejemplo: aumentan las informaciones triviales, pero el verdadero conocimiento se reduce y se concentra en pocos lugares y en pocas manos, la diversidad disminuye y se difunde el conformismo, se enfatiza la creatividad pero se educa a la mediocridad globalizada. Por esto la solución del problema no está al alcance de la técnica, y apela a las ciencias humanas, al arte y a la comunicación.

En este artículo la relación entre las TIC, la sobreproducción y los problemas de la sociedad de la información se explicará, de acuerdo a este enfoque humanista, verificando tres hipótesis: que ciertas propiedades de los medios digitales favorecen el subdesarrollo cognitivo; que el exceso y la sobreproducción cognitiva producen, contrariamente a la retórica de los monopolios digitales, la sociedad de la ignorancia; que el telos nihilista posmoderno no cultiva la creatividad y la diversidad, sino la repetición y el conformismo. Para esto, comenzaremos analizando la relación entre la sobreproducción y la ignorancia; sucesivamente trataremos de explicar qué propiedades de los procesos tecnológicos están involucradas en dicho proceso, luego analizaremos sus efectos en la esfera cognitiva y, por último, discutiremos una alternativa ontológica al actual paradigma tecnológico.

\section{El exceso, las TIC y la sociedad de la ignorancia}

Los efectos del exceso de datos, conceptos, saberes y mensajes requieren 
nuevas modalidades de gestión del saber porque alteran los aspectos cognitivos de la sociedad globalizada. A través del concepto de sociedad de la ignorancia, Kurz y Vega Cantor han desarrollado una argumentación que con el pasar de los años se demuestra, al respecto, cada vez más incisiva:

Siguiendo con la lógica mecánica de la inteligencia artificial, en la era de la información el saber se puede expresar en la ecuación: tecnología + cantidad de información = conocimiento. Los términos de esta ecuación expresan claramente a lo que se reduce el conocimiento en estos momentos: al empleo de tecnologías que aceleran el procesamiento de información, las cuales generan un gran cúmulo de datos, cuya cantidad supera la capacidad de procesamiento individual de una persona, sin que eso signifique en verdad conocimiento, entendiéndolo como producto de la acción de pensar, de reflexionar o de teorizar(Vega, 2007).

El efecto de la sobreproducción, entonces, es ahogar la comunicación en la trivialidad, lo que pasa desapercibido porque la tecnología ofrece efectivamente las herramientas para dominar el exceso y la trivialidad, y con esto hace olvidar que el verdadero saber sigue escaso y en manos de pocos.

Por otro lado, se trataría también de averiguar la verdadera dinámica de esta abundancia de informaciones y conocimientos. En realidad, el capital cognitivo de la sociedad se devalúa porque los usuarios se conforman con manejar herramientas y aplicaciones cuyos criterios y lógicas informáticas no son suyos:

En sentido estricto, información no es conocimiento, cuando mucho conocimiento trivial, similar a estar enterado del movimiento de la bolsa de valores o del momento en el que llega el próximo bus a la estación de Transmilenio. Cuando se mezclan como sinónimos conocimiento e información en realidad están en juego dos categorías de conocimiento: el de las señales y el funcional. Este último está reservado a la elite tecnológica "que construye, edifica y mantiene en funcionamiento los sistemas de aquellos materiales y máquinas 'inteligentes'". El conocimiento de las señales, por el contrario, compete a las máquinas, pero también a sus usuarios, por no decir a sus objetos humanos (Vega, 2007).

En síntesis, si bien es difícil negar que el exceso de herramientas e informaciones reduce la calidad cognitiva de la sociedad, queda por explicar el cómo y el porqué. Ciertamente, hay unos mecanismos de las tecnologías de la información a los que podemos echar parte de la culpa de esta situación; sin embargo, los problemas son más profundos y anteriores a lo tecnológico, ya que se hunden en la crisis metafísica de la posmodernidad.

Si volvemos a leer As we may think desde esta perspectiva, se evidencia el enlace de la tecnociencia con ciertos temas de la filosofía y de la literatura, por ejemplo, la "condición posmoderna" de Lyotard (1987), lo "efímero" de Vattimo (2000) y los "bárbaros" de Baricco (2008). Lo que se delinea en estos ejem- 
plos es un saber antimetafísico, variado y efímero, que mezcla teorías, culturas y lenguajes en el flujo de un relativismo creativo y liberador que responde al telos posmoderno, un nuevo saber sobre el saber, un entretejido de relaciones, de enlaces y conexiones en tiempo real. ¿Pero, es efectivamente así?

En primer lugar, la fragmentación y lo efímero no eliminan las influencias de la metafísica y de los grandes relatos, porque estos simplemente fluyen con las modas y se reciclan en cada fragmento y rizomas. Es que no es posible eliminar una milenaria tradición filosófica ignorando su problema, porque así seguimos dependiendo de sus fundamentos, embebidos en el lenguaje, en la educación y en las instituciones; y no es tan fácil desecharse del saber vertical y especializado porque el saber del enlace (la red) es posible solo a partir del conocimiento de sus nodos, de sus raíces y de sus fundamentos esenciales. En segundo lugar, lo efímero no resuelve el problema del exceso; el devenir de las modas hace que los nuevos productos y mensajes destruyan o sustituyan a los viejos, pero si bien no dejan rastros materiales evidentes, a largo plazo sus huellas imperceptibles se acumulan en los hábitos sociales y en los procesos cognitivos.
Ahora, el problema de los medios digitales es que permiten al usuario de los sistemas informáticos superar el asunto de los fundamentos, pues el marco aplicativo de las interfaces no le abre esta clase de cuestiones. Y este mismo usuario puede también operar con efectividad en la superficie del saber, lo que en términos informáticos se traduce en navegar (operar al nivel de la interfaz).

Entonces, hay una sinergia entre las cuestiones filosóficas irresueltas y las propiedades de los medios digitales, precisamente porque la metafísica opera en el lenguaje y en los saberes embebidos en el software; dicho de otra manera, el usuario no se da cuenta de estar en una jaula metafísica porque las interfaces interfieren entre el usuario y el software y no permiten reconocer los fundamentos del código subyacente.

Las herramientas digitales, en efecto, pueden ser leídas como una metáfora de la condición posmoderna. En el siguiente párrafo trataremos de explicar la relación entre los efectos de la industria cultural y los mecanismos de la tecnología digital, precisamente aquellos que no obstante permanecer ocultos ${ }^{1}$, transforman la sociedad del conocimiento en la sociedad de la ignorancia.

1 Una consecuencia de este ocultamiento es que muy a menudo los new media studies confunden las propiedades de las aplicaciones con las propiedades estructurales del medio digital en cuanto tal. Las aplicaciones, pues, son contextos regidos por sus propias reglas y con sus propios problemas, son una vista o una interpretación determinada que cambia 


\section{Efectos y desperfectos de la sobreabundancia cultural y tecnológica}

El exceso material o cultural es esencialmente la consecuencia de dos procesos: la acumulación o la sobreproducción. En el primer caso se excede al guardar informaciones hasta superar la capacidad de la memoria o del razonamiento; en el segundo caso, al crear más de lo que se puede ver, leer o escuchar. En ambas situaciones hay elementos en común que es preciso tomar en cuenta: el momento histórico de la globalización, la lógica económica de la industria cultural, el problema del arte y de la creatividad y la tecnología. El punto es que la teoría crítica no avanza mucho porque no relaciona lo tecnológico con los demás aspectos del problema, y sobre todo, porque no trata de averiguar cómo sus cuestiones ontológicas afectan a los procesos cognitivos.

\section{La globalización}

La globalización, última etapa del desarrollo económico contemporáneo, es una dimensión que incentiva la sobreproducción cultural y material, porque abre a las fuerzas productivas mercados nuevos y uniformados en cuanto a hábitos y gustos, lo que evidentemente mejora las oportunidades de distribución.

El hecho de uniformar los gustos y las modas a través del imperialismo cultural y de los nuevos medios de comunicación oculta la existencia del exceso y de la saturación, porque facilita el consumismo acrítico y globalizado.

\section{La sobreproducción}

El exceso y la saturación son fenómenos recientes, pues se deben al crecimiento demográfico, al bienestar y a la relativa difusión de la democracia. En este contexto, la industria cultural, que prospera solo si hay consumo, debe proponer continuamente nuevos productos, modas y géneros artísticos para mantener viva la atención de un público irremediablemente distraído, víctima del marketing y del escándalo y enviciado por la lógica del entretenimiento y del espectáculo (Vargas Llosa, 2012).

\section{El problema del arte y de la creatividad}

Los artistas, al comienzo de la modernidad, percibieron con claridad el agotamiento de los medios creativos y expresivos; la historia del arte, al fin

continuamente y que no satura las posibilidades del medio digital. Además, los procesos cognitivos (científicos o artísticos), implementados en los algoritmos, son mediados por las interfaces, que no permiten ver sus estructuras esenciales. Dicho de otra manera, sería como construir una filosofía de la ciencia considerando solamente los errores de los manuales de química. 
y al cabo, concluye con la liberación completa del arte, que en realidad cierra el horizonte de sus posibilidades lingüísticas y formales (Belting, 1990). Personajes literarios como Bouvard y Pecuchet de Flaubert (1999), el compositor Leverkühn de Mann (1998), el magister ludi de Hesse (1949) y el nihilismo de vanguardias como el dadaísmo o el Pop Art son ejemplos muy claros de este estado crítico del artista contemporáneo. Se trata obviamente de una dinámica irreversible, y hay que ser insensibles o distraídos (muchos lo son) para no tomar en cuenta este fenómeno en la lectura de los medios de comunicación, analógicos o digitales, que solamente han traducido a la cultura de masa los problemas puestos por intelectuales como Flaubert, Mann y Hesse.

\section{La tecnología}

La sobreproducción es facilitada por la eficiencia de las herramientas digitales (desde el software aplicativo a la robótica) y por la velocidad y precisión de la distribución (los medios de comunicación masivos y sobre todo Internet). El lenguaje digital, además, produce exceso porque al alimentar el gusto por la complejidad tecnológica crea la necesidad de otras tecnologías destinadas a simplificarla, lo cual, paradójicamente, genera nueva complejidad (piénsese en los virus informáticos y el mercado de los antivirus). De esta forma, la comprensión de los procesos tecnológicos se vuelve cada vez más difícil y se condena a los usuarios a la superficialidad.

\section{Desde el exceso al subdesarrollo cognitivo}

La sobreproducción cultural y tecnológica genera entonces excesos que no se pueden manejar correctamente, y esto afecta los procesos epistemológicos, críticos y creativos. En seguida se examinarán los daños cognitivos que pueden producir las dinámicas anteriormente analizadas, dentro de los procesos tecnológicos y en el umbral ontológico posmoderno que hemos establecido como marco teórico.

\section{Conservadurismo}

La actitud conservadora se impone por tres razones. La primera es el mecanismo del mercado masivo, que no quiere innovación y experimentación; para absorber la sobreproducción, pues, es necesario que el público quiera siempre lo mismo, esto es, lo que conoce. El segundo aspecto se debe a la naturaleza de las aplicaciones digitales: cualquier software aplicativo se basa en la simulación de técnicas analógicas tradicionales ${ }^{2}$, y los programadores no pueden crear procesos digitales originales, ya que el

2 Un ejemplo son los programas de fotorretoque, que se basan en los procesos fotográficos en el cuarto oscuro tradicional y en la herencia estética de los fotógrafos pictorialistas de comienzos del siglo XX, como Coburn o Demachy. 
público no los entendería y no sabría cómo utilizarlos ${ }^{3}$. El problema del conservadurismo digital se debe también a la backward compatibility (Raskin, 2003): estos términos significan que las interfaces de las nuevas soluciones informáticas deben tomar en cuenta los hábitos y los conocimientos establecidos de los usuarios, lo que hace imposible saltos de paradigmas en la evolución tecnológica. $\mathrm{Al}$ respecto, hay que considerar que el usuario promedio es conservador, no desea aprender continuamente nuevas interfaces, porque quiere que su tiempo se concentre en el trabajo. Pero así los usuarios aceptan el statu quo de sus herramientas, cuyas ideas y algoritmos se imponen como normas universales, lo que contrasta con la diversidad y la posibilidad de experimentar procesos alternativos.

\section{Uniformidad y monopolios}

Los procesos productivos de las economías globalizadas se integran mediante estándares para la producción, la distribución y el consumo: un mismo idioma, los mismos parámetros y criterios unificados, entre otros. En el escenario de las herramientas digitales, esta integración implica que se utilicen solo algunas aplicaciones, por ejemplo Photoshop en el campo de la edición de imágenes, Word en la escritura, Google en los buscadores, Facebook en las redes sociales, los protocolos como $\mathrm{TCP} / \mathrm{IP}^{4}$ en las redes de computadoras. Sin embargo:

Another problem with software natural monopolies is the suffocation of innovation which would accompany it. [...] A company with a monopoly, natural or otherwise, has little incentive to improve its product. Only the possibility of real competition can keep a software company on its proverbial toes (Conigliaro, 1996).

La eficiencia de la producción, en lugar de diversificar la disponibilidad de herramientas, acaba reduciendo la tecnología dentro de unos pocos grandes sistemas, cuyo desarrollo requiere inversiones enormes. Por eso las grandes compañías son las únicas que, apode-

3 Todos los programas que han tratado de desarrollar nuevos procesos o interfaces para el diseño, el fotorretoque, etc., no han tenido la difusión esperada entre los profesionales. Hay ejemplos clamorosos del fracaso de productos originales marginados por esta nueva forma de academia, como el software Painter, que hace años se pensaba como una posible alternativa al dominio de Photoshop.

4 La facilidad de uso y los intereses de las grandes compañías hicieron caer el OSI, a pesar de su apertura y mejor arquitectura.

5 “Otro problema de los monopolios del software es que estos necesitan controlar la innovación. (...) Un monopolio no tiene reales incentivos para innovar. Solo un verdadero libre mercado puede mantener en alerta constante una compañía de software" (traducción del autor). 
rándose del mercado y del saber, pueden controlar el futuro de las tecnologías de la información. Pero esto gene- ra un contexto demasiado homogéneo y uniforme, donde la creatividad y la investigación son cada vez menos libres.

\section{Porcentajes de descargas de fotografías de las redes sociales}

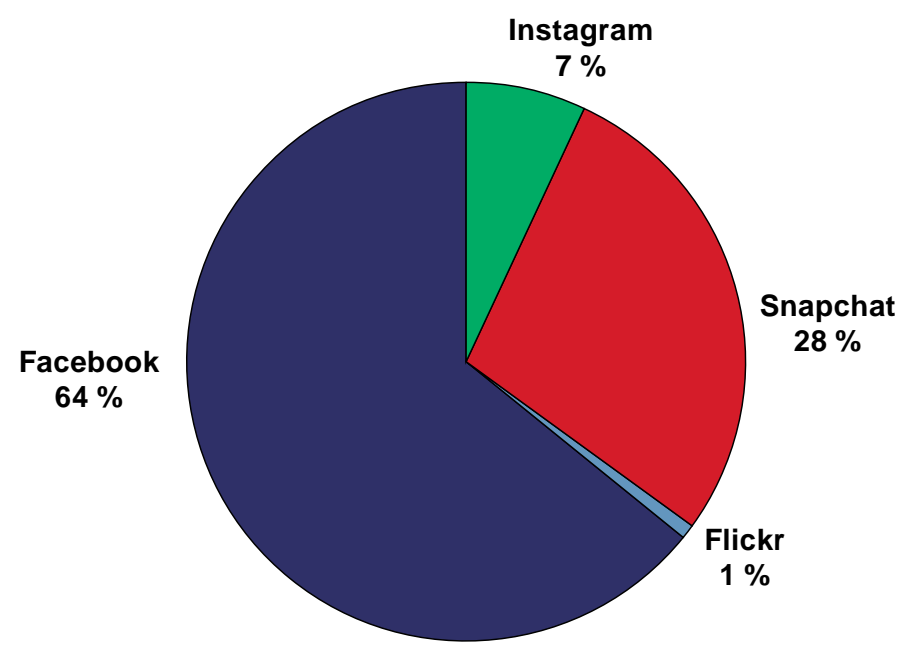

Fuente: BI Intelligence, 2013.

Otro aspecto del gigantismo informático, que tiene como efecto la uniformidad y la homogeneidad, es el interés de las compañías de software por crear aplicaciones multifuncionales que cierran los nichos aplicativos que justificaban la oferta de productos especializados ${ }^{6}$. Por otro lado, la creciente complejidad funcional que se requiere del software, como la programación de videojuegos, hace que los desarrolladores utilicen sistemas operativos como Windows (Conigliaro, 1996) y aplicaciones middleware que facilitan su desarrollo, por ejemplo, game engines como Frosbyte ${ }^{7}$, programas muy complejos de los cuales hay solo unos pocos en el mercado; de esta

6 Un ejemplo es InDesign, que ofrece todas las funciones que antes eran exclusivas de programas especializados.

7 Frosbyte es un motor de juegos por computadora que se está difundiendo en el mercado por su calidad visual, como los juegos Battlefield y Mass Effect (véase http://www.frostbite.com). 


\section{Porcentajes de mercado del software para la edición de video}

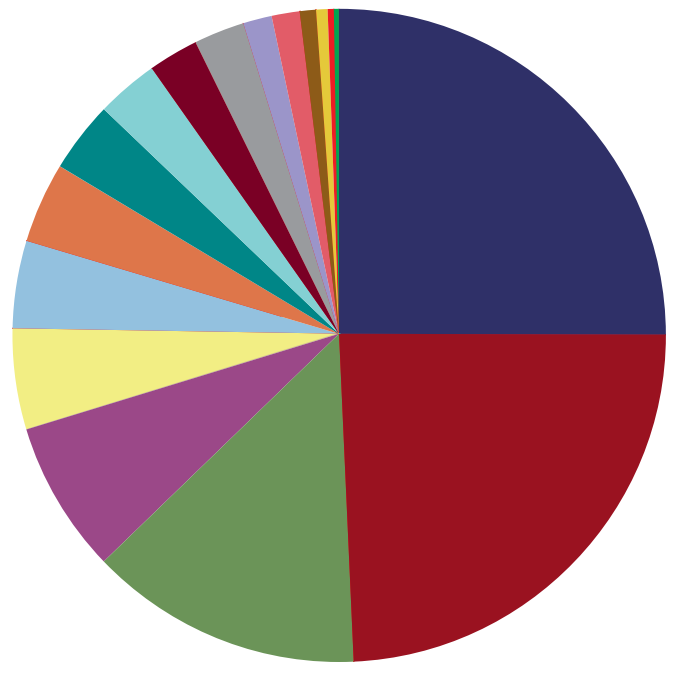

Fuente: NLE Survey 2014.
Adobe Premiere Pro CS6

Adobe Premiere Pro CC

Apple Final Cut P ro X

Sony Vegas Pro 12

Adobe Premiere Pro CS5.5

Apple Final Cut Pro 7

Adobe Premiere Pro CS5.5

Sony Vegas Pro 11

Apple iMovie

- Avid Media Composer 7

Adobe Premiere Elements

Adobe Premiere Pro CS4

Sony Vegas 12

- Cyberlink Powerdirector 10

Lightworks NLE

Hitfilm 2 Ultimate

Final Cut Pro 6

Avid Newscutter

Seven Grass Valley Edius

OpenShot

Windows Live Movie Maker forma, los productos informáticos se asemejan todos, como sucede, además, en otros campos de la industria.

Ahora bien, se podría objetar diciendo que uniformar tiene ventajas innegables: un solo ambiente de desarrollo hace que las energías y los recursos no se desperdicien, permite compartirlos con mayor facilidad y las librerías unificadas proveen funciones que aceleran y simplifican el desarrollo de varias aplicaciones. Estas facilidades ayudan sobre todo a los pequeños desarrolladores, recortando sus tiempos, y hacen a las TIC más estables y seguras, pues sabiendo que los estándares no van a cambiar de un día pa- ra el otro, minimizan los riesgos de la inversión. Entonces, los monopolios y la uniformidad son un pequeño precio que habría que pagar para aprovechar estos servicios esenciales.

Todo esto es cierto, pero no anula el hecho de que se produzca la uniformización de conceptos, procesos y herramientas, y esto refuerza al sistema monopolístico, ya que la moneda con que se paga la eficiencia es la creatividad de los usuarios.

\section{Conformismo}

La estandarización de las TIC se traduce en el conformismo de sus usuarios. 
Nótese que el conformismo es diferente de la uniformidad, pues tiene todo el sentido negativo de un comportamiento pasivo y sin crítica. De todos modos, es un fenómeno que se produce por dos razones.

Desde el punto de vista de la industria cultural, sus mecanismos efímeros generan conformismo, pues su libertad estética y conceptual es aparente: al ignorar el fundamento metafísico, todos los mensajes adoptan implícitamente el existente, esto es, el romántico moderno que lo efímero pretende negar. Lo efímero es como la noche de Hegel, donde todas las vacas son grises.

Desde el punto de vista de los usuarios, el uso compartido y masivo de pocos lenguajes y herramientas digitales pone en minoría todo lo demás, cuyos usuarios se quedan con problemas de comunicación y compatibilidad; obviamente, de esta forma se produce el conformismo cultural y social, pues a nadie le gusta estar marginado. El problema del conformismo ha sido estudiado recientemente por el Pew Research Center (Hampton, Rianie, y Purcell, 2014), comprobándose que los ususarios de las redes sociales tienden a eliminar las opiniones que generan comentarios desfa- vorables, y que si el usuario se percata de que su opinión no es compartida, es más rehacio a expresarla espontáneamente. Esto traduce a los medios digitales el fenómeno de la "espiral del silencio", propuesta en los años setenta por la socióloga Noelle-Neumann (1993) ${ }^{8}$.

\section{Atrofia cognitiva}

El prototipo crítico a las tecnologías de la comunicación es la crítica a la escritura de Platón. Generalizando, el argumento es que la tecnología debilita la función física o intelectual que pretende apoyar, como la memoria en el caso de la escritura; Rousseau o McLuhan, aunque utilizando diferentes términos (el suplemento, la extensión del órgano, etc.), en efecto, no hacen más que reinterpretar el original razonamiento platónico. Con respecto a los medios digitales, el daño puede ser más notorio, no solamente porque estas herramientas, que se presentan como extensiones del cerebro (la memoria, el cálculo), ofrecen procesos con un alto grado de automatización que delegan al software muchos aspectos de la gestión de los conocimientos (embebidos en los algoritmos) y de la

8 Sin embargo, hay opiniones contrarias: no hay silencio sino fragmentación y creación de pequeñas islas de consenso. Pero simplemente se mueven la globalización y el conformismo en otro nivel, pues lo que genera conformismo será la isla mayor. Paradojicamente, además, hay el fenómeno de la espiral del silencio dentro de la misma isla de consenso con respecto a sus miembros de referencia. Se forma una opinión pública diversificada pero parcelada, jardines cerrados, el walled garden, como decía Tim Berners Lee a propósito del Facebook. 
labor cognitiva, sino también por su carácter adictivo ${ }^{9}$. A la larga, es inevitable que todo esto degenere en una suerte de flojera cognitiva y en la aceptación pasiva de la ignorancia.

\section{La crisis de la creatividad y la apatía}

Desde el punto de vista de la estética, podríamos sintetizar el resultado de todas estas dinámicas como el "efecto Bouvard y Pecuchet", en homenaje a los copistas del relato de Flaubert (1999). La historia de estos dos empleados, que renuncian a un empleo seguro para convertirse en escritores, es la alegoría de la crisis de los artistas modernos que chocan, sin darse cuenta, contra la triste realidad de que todo está hecho, o requiere un trabajo tan pormenorizado que termina quitando entusiasmo y energía creativa. Es así que Bouvard y Pecuchet renuncian a cualquier ambición artística y se liberan de su angustia estética volviendo a los simples placeres que les da la labor de copistas.

Ahora, los artistas posmodernos pretenden resolver la crisis dramatizando el efecto Bouvard y Pecuchet, a través de una suerte de mise en abyme del mismo problema de la creativi- dad, con prácticas como el reciclaje, la apropiación y el collage $e^{10}$, como se declara en el manifiesto de la posfotografía (Fontcuberta, 2011). Pero semejantes procesos combinatorios $\mathrm{u}$ autorreferenciales, claro está, no son auténtica poiesis, porque se fundan sobre mecanismos azarosos, esto es, no cuentan con el telos o el élan vital que mueve una creación artística original.

El punto es que el efecto Bouvard y Pecuchet, potenciado por la eficiencia de las tecnologías de la información, produce una angustia estética mucho más dramática, pues los artistas de hoy, copistas prisioneros de los simulacros y de las interfaces, ni siquiera se percatan del problema ${ }^{11}$.

\section{Ontología y austeridad tecnológica}

De acuerdo a lo que hemos visto, la sociedad del conocimiento es una ficción o una metáfora que no funcionan, y ciertamente los mecanismos digitales son parte de esta distopía cognitiva en la que se está convirtiendo la sociedad contemporánea. Los problemas de la sociedad de la información se deben enfrentar desde varios puntos de vis-

9 Tanto, que se llega al absurdo de ciertas simulaciones que tanto simulan que no se dan cuenta que están inventando el agua caliente, es decir, implementando funciones computarizadas iguales a las que el cuerpo hace por su propia naturaleza (el caso de la interfaz Sixth sense del MIT).

10 En efecto, el exceso de disponibilidad de conceptos, herramientas y materiales hace proliferar la costumbre de la copia, de hacer cosas con piezas de otras cosas.

11 Pues nadie es más conformista que quien se cree original y creativo. 
ta, pero las cuestiones filosóficas y ontológicas son primordiales para poder corregir el uso y el abuso de las herramientas digitales. Como enseñó Heidegger (1977), la tecnología es el telos y la lógica de la técnica; si esto es cierto, para corregir la tecnología una intervención solamente técnica no haría sino aumentar el problema (la sobreproducción, el exceso y sus consecuencias cognitivas). Lo que hay que hacer es cuestionar los fundamentos ontológicos de las TIC, en otras palabras, actualizar nuestra lista de prioridades y estrategias culturales, precisamente lo que trataremos de hacer, a modo de conclusión, en los siguientes acápites.

\section{La profundidad y la necesidad de la alta cultura}

La primera posibilidad que se discute (Lipovetsky, 2003; Baricco, 2008) es que la cultura light (el conformismo, la superficialidad, el espectáculo) podría constituir la forma que necesariamente debe asumir la cultura en la posmodernidad, lo que podría elaborar nuevos valores, pues la cultura light, según este punto de vista, ha sido capaz de regalar a las masas más democracia y libertad cultural.

Pero la cultura light no abre horizontes de significados originales y no crea los nuevos valores que la posmodernidad necesita, porque solo recicla los saberes existentes. Y si eventualmente de nuevos valores y saberes se tratara, igualmente no estaríamos autorizados a considerar superada la cultura clási- ca, como pretenden los nuevos bárbaros de Baricco, más bien, lo light podría ser una alternativa, algo más que tenemos que aprender. Por eso el verdadero nuevo saber será algo muy complejo: la superficie y el vacío, la red más el nodo, el rizoma y el árbol.

Ahora, el dominio de un contexto epistemológico superior requiere capacidades y entrenamientos exclusivos, con lo cual necesariamente se diseñarán nuevas jerarquías sociales y culturales y se profundizarán las brechas cognitivas y tecnológicas: sin las necesarias acciones educativas y tecnológicas, los seudoconocimientos y las banalidades de la cultura light aumentarán progresivamente, mientras la creatividad y los saberes verdaderos serán prerrogativa de elites cada vez más excluyentes.

Sin embargo, aquí hay que hacer una anotación importante, es decir, que también los conocimientos de orden superior resultan en exceso, y que por esto los monopolios deben necesariamente seleccionar los saberes y reducir su diversidad (precisamente para que puedan ser monopolizados). Así que la complejidad del saber debe defenderse políticamente, en el nivel de decisiones estratégicas corporativas e institucionales. La alta cultura es la única que puede asumir semejante tarea.

\section{Glocalización}

Con respecto a la relación entre exceso, uniformidad y globalización, una propuesta que pone de acuerdo a muchos teóricos y críticos de la posmodernidad 
(Latouche, 2009; Friedman, 2003) es el regreso a las dimensiones locales, a los sistemas pequeños, a las islas de producción y comunicación autosuficientes en el mar de la globalización. Dicho discurso se sustenta argumentando que el saber y la comunicación pierden su sentido y su originalidad cuando están descontextualizados o dentro de sistemas demasiado grandes; el significado verdadero de la innovación se da en el contexto local, pues es algo nuevo con relación a una situación sociocultural determinada, y en un marco de necesidades específicas.

Por otro lado, la fusión, hija del aburrimiento globalizado y del cut and paste, es repetición disfrazada y conformismo maquillado precisamente porque olvida la relación con el origen (con el hic et nunc) de sus elementos. Recuperar lo auténtico dentro la fusión implica, entonces, liberarse de las modas, de los idiomas dominantes, de los monopolios culturales, de los gadgets digitales y de los modelos educativos estandarizados.

\section{Lo real, la oralidad y la interactividad}

Otra estrategia de lucha cultural podría ser el regreso a lo analógico, a la materia y al cuerpo, abandonando lo virtual por lo real y lo artificial por lo natural. En términos comunicacionales, se tra- taría de renunciar a los discursos tecnológicos para volver a la comunicación natural, es decir, a la oralidad. El mensaje oral, que es inmaterial e inmediato, se consuma en tiempo real y no deja excesos; sin embargo, es efectivo y significativo (no es superficial) porque se hunde en el físico y en las emociones (Ong, 2000). Además, según Ong, los medios digitales, por su velocidad e interactividad, tendrían las mismas propiedades de la comunicación oral. Sin embargo, en las TIC todo es escritura, aunque parecido a la oralidad: el software es escritura, la multimedia es escritura, el chat es escritura ${ }^{12}$; en este sentido, la comunicación digital no es efímera como la oralidad sino sólida, persistente y contaminante.

Ahora, para controlar los daños cognitivos de los medios digitales, un número cada vez mayor de educadores, teóricos y padres de familia pide restringir el uso de los gadgets; pero se sabe que el prohibicionismo nunca ha dado los resultados esperados, entonces hay que buscar una alternativa.

La solución se podría hallar a partir de la misma naturaleza de las TIC, que es la de las metaformas: herramientas para hacer herramientas, aplicaciones para hacer aplicaciones (como, por ejemplo, los lenguajes de programación). Las TIC de esta clase nunca están

12 Para una crítica a la segunda oralidad de Ong véase mi libro Filosofía y software. La cultura digital detrás de la pantalla (2012). 
en exceso ${ }^{13}$ y no generan conformismo porque son procesos creativos y heurísticos. Todo esto se explica más a través del concepto de caritas y analizando la relación entre la dimensión privada y la pública de la producción cognitiva y de la comunicación, como veremos enseguida.

\section{La caritas}

El exceso, la sobreproducción y sus efectos negativos se pueden corregir de dos maneras. En primer lugar, reduciendo el consumo material e intelectual, o de acuerdo a la propuesta de la gift economy (Cheal, 1988), compartiendo lo que nos sobra y regalando lo que no necesitamos. Al respecto, se podría objetar que compartir y regalar tienen sentido en un contexto de austeridad y de escasez, no de abundancia. Sin embargo, desde el punto de vista epistemológico, la escasez se refiere a los criterios y al pensamiento crítico.

En segundo lugar, la sobreproducción se corrige controlando el impulso creativo y productivo (la poiesis). Aunque también se podría objetar que así se penaliza la creatividad y el libre pensamiento. Pero la poiesis podría manifestarse libremente, es decir, sin producir excesos, si no se justifica por interés o por vanidad.

En ambos casos nos encontramos con la ontología de la caritas, que evidentemente no consiste en la división de los bienes materiales o de los conocimientos en sí, sino en la distribución de procesos cognitivos abiertos para la creación y la gestión de los conocimientos: las metaformas ${ }^{14}$. Una cultura tecnológica orientada a la caritas hace que los recursos cognitivos no sean monopolizados por las élites económicas o intelectuales ${ }^{15}$.

\section{Individuo y colectividad}

Para redondear el concepto de caritas, en lo que se refiere a los problemas cognitivos de las TIC, analizaremos las dimensiones pública y privada de la comunicación y de la creatividad. En síntesis, lo que realmente cuenta son los procesos públicos, es decir, las herramientas cognitivas abiertas que pueden ser utilizadas por muchos (como es el caso de las metaformas); estas se encuentran constitutivamente en un estado de real escasez, pues los cambios económicos, políticos y sociales requieren que el ingenio humano adecúe siempre sus instrumentos

13 Pues el exceso depende de lo que hace el usuario con dichas herramientas, esto es, algo que se decide en el ámbito individual: un caso explicativo es lo que sucede con los makers y la impresión 3D, una aparente revolución que, salvo algunas excepciones, servirá para llenarse de cachivaches de filamento ABS.

14 El software libre, el copy left y el open source son aspectos de la caritas tecnológica.

15 Internet y el ciberespacio como utopías realizables. 
intelectuales, lo que muy pocos harán. Entonces, puesto que no todos tienen la capacidad para generar contenidos innovadores en tiempo real, los que se logren hacer deben ser compartidos con justicia ${ }^{16}$.

Finalmente, la caritas se justifica cognitivamente porque su contribución no es material, sino conceptual; es una herramienta que puede servir para hacer limpieza, lo que se puede entender como una nueva manera de crear. Por lo menos, esto ya no depende de las tecnologías digitales, sino de las intenciones de cada individuo en cuanto creador y comunicador.

\section{Referencias}

Baricco, A. (2008). Los bárbaros. Ensayo sobre la mutación. Barcelona: Anagrama.

Belting, H. (1990). La fine della storia dell'arte o la libertà dell'arte. Turín: Einaudi.

Bush, V. (Julio de 1945). As we may think. Atlantic Monthly, 12. Boston: Houghton Mifflin.

Carr, N. (2008). Is Google making us stupid? What the Internet is doing to our brains. Recuperado de http://www. theatlantic.com/magazine/archive/2008/07/is-google-making-usstupid/306868/
Cheal, D. J. (1988). The gift economy. Nueva York: Routledge.

Conigliaro, A. (1996). The dangers of computer monopolies. Recuperado de http://cs.stanford.edu/people/eroberts/cs201/projects/corporate-monopolies/index.html

Fishwick, P. A. (Ed.). (2008). Aesthetic computing. Cambridge: The MIT Press.

Flaubert, G. (1999). Bouvard et Pécuchet. Barcelona: Tusquets.

Flusser, V. (2007). Towards a philosophy of photography. Londres: Reaktion Books.

Fontcuberta, J. (11 de mayo de 2011). Por un manifiesto posfotográfico. La Vanguardia.

Friedman, Y. (2003). Utopie realizzabili. Macerata: Quodlibet.

Hampton, K., Rainie, L., y Purcell, K. (2014). Social media and the 'spiral of silence'. Recuperado de http://www. pewresearch.org/

Heidegger, M. (1977). The question concerning technology. Nueva York: Harper and Row.

Hesse, H. (1949). El juego de abalorios: ensayo de una biografía del "magister ludi" Josef Knecht, juntamente con los escritos dejados por él. Buenos Aires: Santiago Rueda.

Kurz, R. (2003). La ignorancia de la sociedad del conocimiento. Antro-

16 Por el contrario, el producto (el objeto, el mensaje, la imagen o el concepto en sí) se acumula y genera exceso, contaminación y, como hemos visto, daños cognitivos. 
posmoderno [página web]. Recuperado de http://www.antroposmoder no.com/antro-articulo.php?id_ articulo $=247$

Latouche, S. (2009). Farewell to growth. Cambridge: Polity Press.

Lipovetsky, G. (2003). La era del vacío: ensayos sobre el individualismo contemporáneo. Barcelona: Anagrama.

Lyotard, J.-F. (1987). La condición posmoderna, informe sobre el saber. Madrid: Cátedra.

Mann, T. (1998). Doktor Faustus. Vida del compositor alemán Adrian Leverkühn narrada por un amigo. Barcelona: Edhasa.

Noelle-Neumann, E. (1993). The spiral of silence: public opinion - our social skin. Chicago: University of Chicago.
Ong, W. (2000). Orality and literacy. Nueva York: Routledge.

Raskin, J. (2003). Interfacce a misura d'uomo. Milán: Apogeo.

Vattimo, G. (2000). La società trasparente. Milán: Garzanti.

Roncoroni, U. (2012). Filosofía y software. La cultura detrás de la pantalla. Lima: Universidad de Lima, Fondo Editorial.

Vargas Llosa, M. (2012). La civilización del espectáculo. Madrid: Alfaguara.

Vega Cantor, R. (Junio de 2007). La sociedad del conocimiento: una falacia comercial del capitalismo contemporáneo. Herramienta, 35. Buenos Aires: Ediciones Herramienta. 